\title{
Modeling of Powder Behavior. Report for Phase 2 Feasibility
}

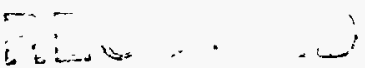

FEg 20 HS

K. Sinz

OSTI

D. H. Lassila

D. W. Baum

January 11, 1996

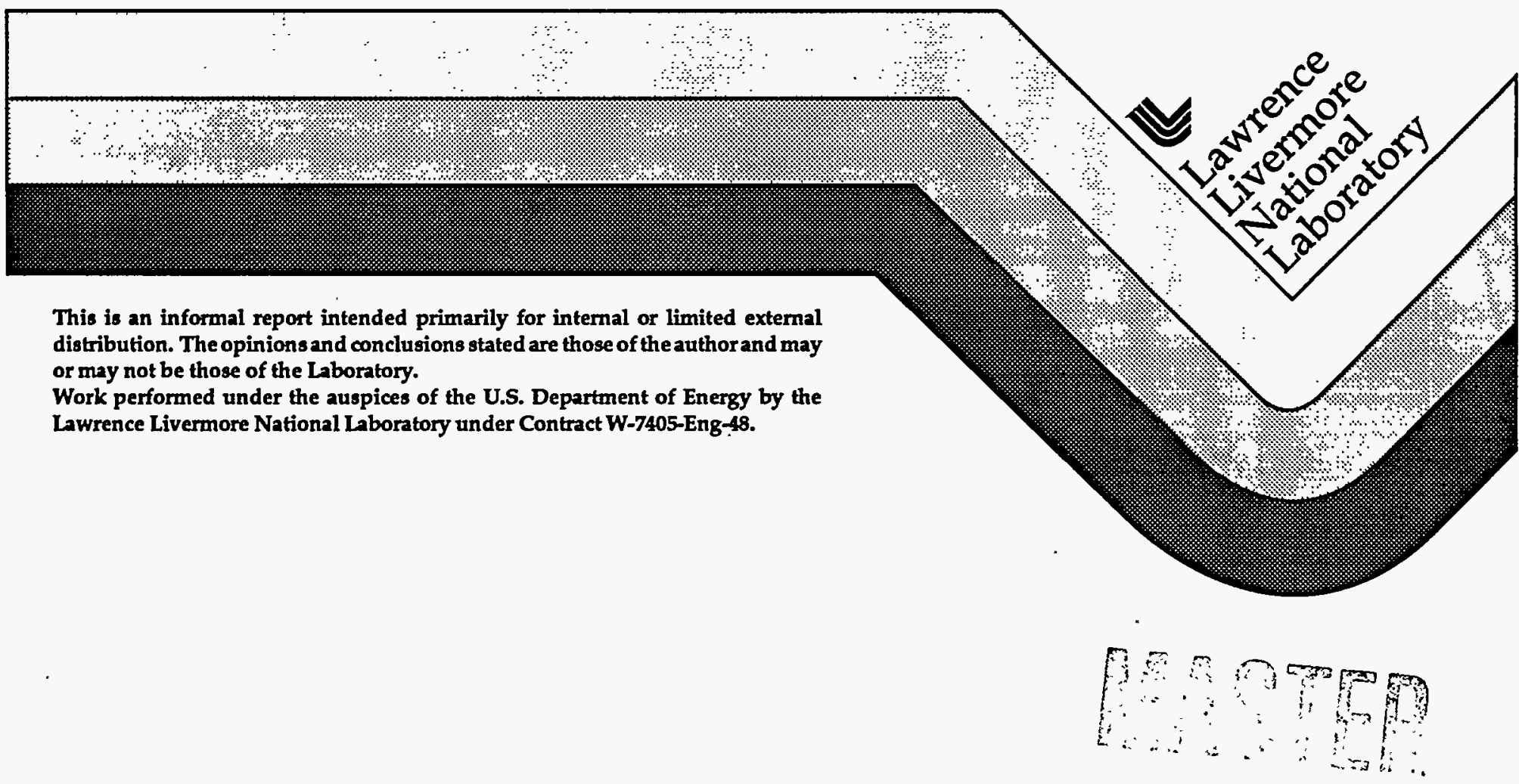

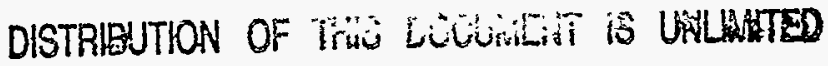




\section{DISCLAIMER}

This document was prepared as an account of work sponsored by an agency of the United States Government. Neither the United States Government nor the University of California nor any of their employees, makes any warranty, express or implied, or 2ssumes any legal liability or responsibility for the accuracy, completeness, or usefulness of any information, apparatus, product, or process disclosed, or represents that its use would not infringe privately owned rights. Reference herein to any specific commercial products, process, or service by trade name, trademark, manufacturer, or otherwise, does not necessarily constitute or imply its endorsement, recommendation, or favoring by the United States Goverament or the University of California. The viewsand opinions of authors expressed herein do not necessarily state or reflect those of the United States Government or the University of California, and shall not be used for advertising or product endorsement purposes.

This report has been reproduced directly from the best available copy.

Available to DOE and DOE contractors from the Office of Scientific and Technical Information

P.O. Box 62, Oak Ridge, TN 37831

Prices available from (615) 576-8401, FTS 626-8401

Available to the public from the National Technical Information Service

U.S. Department of Commerce 5285 Port Royal Rd., Springfield, VA 22161 


\title{
Modeling of Powder Behavior: \\ Report for Phase 2 \\ Feasibility
}

\author{
Kurt Sinz, David H. Lassila, and Dennis W. Baum
}

11 January 1996

\author{
Lawrence Livermore National Laboratory \\ P.O. Box 808 \\ Livermore, California, 94551 \\ USA
}

\begin{abstract}
We report on a Phase 2 feasibility study of an effort to compute the mechanical behavior of the incendiary powder RS41 during compaction and release using the experiments conducted at China Lake as a data base. Our simulation, using a prototype material model, develops two-dimensional density gradients in even these simple, uniaxial double-piston experiments. In our view, the computational simulation of the behavior of RS41 while press loading this material into a round and under subsequent launch conditions is feasible within the framework of current technology. For the model development that was conducted as part of this feasibility study, the code of choice was the implicit Lagrangian hydro code NIKE2D. The applicability of the explicit companion code DYNA2D is also discussed.
\end{abstract}

\section{Introduction}

The incendiary powder RS41 has long been used in military munitions. It is a mix of powdered $\mathrm{MgAl}$ alloy, $\mathrm{KClO}_{4}$ grains and a small amount of calcium resinate. The material is used as an incendiary in the nose of munitions ranging from $20 \mathrm{~mm}$ to $40 \mathrm{~mm}$ and perhaps higher calibers. It is desirable to predict computationally the observed material density distribution that results from press loading RS41 into the nose of a round during manufacture. A predictive capability is also needed to calculate the change in the density distribution that results from the acceleration and spinnup of the round in a gun barrel during launch and from in-flight deceleration. This final density distribution is the initial condition of the material before impacting a target and is believed to be important to the round's overall performance, particularly with regard to shock-induced deflagration.

China Lake has conducted compression and release tests on this material and on an inert simulant using the double piston apparatus described by Elban, et. al. (Ref. A). In the current report we attempt to demonstrate the feasibility of

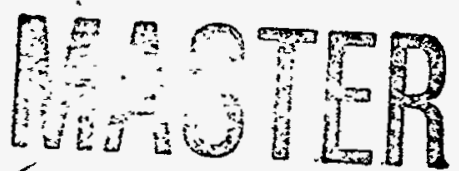


using such data to construct a computer model for the behavior of RS41 for the purpose of calculating the density gradients in rounds under various mechanical conditions.

The effort included an analysis of China Lake's data for consistency and then using this data to construct a prototype material model for RS41 to calculate one of their experiments. This was sufficient to determine that 1) some high quality data for RS41 has been obtained, 2) a suitable material model for RS41 can be constructed for this experiment and 3) that even in this simple experiment, density gradients are found that result from wall friction. Density gradients are expected to be even more prevalent in rounds which have a more complicated geometry with less ideal wall conditions than the laboratory-perfect double-piston apparatus which is made of hardened steel.

\section{Technical Discussion}

\subsection{Description of China Lake Experiments}

China Lake used the double piston experiment to measure some of the mechanical responses of RS4I under loading and release conditions. In this type of experiment, a thick, hollow steel cylinder has two movable steel pistons inserted into it that confine the powder (sample) to be compacted (Fig. 1). This assembly is placed vertically into a compression device. The force applied to the top piston is measured, as is the force transmitted to the bottom piston. The difference is taken up by wall friction between the compacted powder and the steel cylinder. Hoop strain on the outside of the steel cylinder is also measured so that triaxial data are obtained in this experiment for material modeling.

The experiments include six experiments for RS41 (dubbed COMPAC29 COMPAC34) and 4 experiments for simulant (dubbed COMPAC36 COMPAC39). The data includes the loading as well as the release. Several experiments were done that included a reloading and release after the initial release. This latter data was not evaluated in any detail

\subsection{Data Evaluation}

We conducted extensive consistency checks on the data obtained from China Lake. Our attention was limited to the initial loading and unloading of samples of RS41. All inconsistencies in the data were resolved within the current set of experiments through many helpful interactions with China Lake (Ref. B) 
We believe that the resulting data are consistent and of good quality. The one data item that is of distinctly lower quality than the rest are the

measurements of hoop strain on the outside of the cylinder. This data item is possibly of sufficient quality to be useful when special numerical methods are employed to process the data.

Our effort centered on RS41 experiments and we made no comparison between RS4I and simulant. In all cases I attempted to use the primitive data for evaluation. This approach reduced the amount of linearization that is often necessary in data interpretation.

\subsection{Data}

An example of China Lake's data is shown in Fig. 2. The results of three experiments are shown after some amount of inference. The piston displacements are the top curves, while the next highest curves are the applied stresses, and the next highest curves (dashed) are the transmitted stresses. The noisy curves are the radial stresses whose inference will be discussed below. The amount of piston displacement scales directly with the sample volume when the small elastic distortions of the apparatus are neglected. The plateaus on the displacements are then the experimental domain during which the sample is compressed but the volume is held constant before unloading. It is important to note that during this time the stresses - applied and transmitted - on the surfaces of the sample decay. We are not currently modeling this behavior but we believe that it is possible to represent this phenomenology with a model extension to be discussed later.

The precision of this data appears to be very good. Despite the noise in the curves for radial stresses an obvious similarity between these curves and the axial stress curves is apparent. In fact, fitting the average axial stresses to these curves results in plausible fits that eliminate the noise. Unfortunately the inferences that led to the radial stress data were not available to us when the model was formulated and no use was made of this information during the development of the prototype material model for RS41.

Fig. 3 shows a plot of the average axial stresses versus the fractional theoretical maximum densities (fTMD) for all RS41 experiments. It is apparent that the precision is good and that there is no great variation in the slopes of the release curves between the two sets of experiments. Also shown are the fits that were used as the representation of the average of the experimental values.

The average axial stresses that we used were simply the average of the applied and the transmitted axial stresses on the sample. The density of the sample was the mass of the sample divided by the volume of the test chamber. We 
refer to this as a linear method of data interpretation in that it ignores possible internal gradients both in stress and in density. While this is a good starting assumption our analysis shows that internal gradients are in fact substantial and a more sophisticated, iterative data analysis is called for.

\subsection{Material Model}

The basic material model (Ref. C) that we used to represent the data included pore crush with an elastic, perfectly plastic strength model plus wall friction. A simple tensile failure criteria is also available but was not needed for the current pilot study.

Since correct radial stress inferences were not available to us during the model formulation phase of this project, the axial stress was separated into the trace and the deviatoric components. One third of the total axial stress was assumed to be the contribution from the stress deviator with the remainder being assigned to the pressure. The additional assumption, that the material yields everywhere along the loading (crushing) portion of the curve, defines the yield strength at a value of $3 / 2$ the stress deviator. This follows immediately from a resolution of the stress components in slab geometry. The decomposition of the stress during the loading phase is illustrated in Fig. 4. Also shown is the ad hoc extrapolation beyond available data. Such an extrapolation is desirable in the event a portion of the calculation gets into a regime where there is no recorded data. This turns out to be the case as discussed below and stems from the fact that internal gradients are in fact important.

The same splitting of the stresses during loading was also applied to the unloading. A simple linear fit gave a bulk modulus of $18 \mathrm{~kb}$ and a shear modulus of $9 \mathrm{~kb}$ was assumed. These values were treated as constant during the unloading from the crush curve, although in general it is expected that the moduli would change with density. This completes the discussion of the main material model. The details of this model and the code inputs for the MAZE generator and for the NIKE code are documented in the appendix. The steel cylinder and pistons were modeled as simple elastic, perfectly plastic material. Plasticity of the steel is never invoked because of the low stress levels at which these experiments are conducted.

A second set of model parameters was also used that assigned one sixth of the total axial stress to the stress deviator on the crush portion of the curve. The unloading portion of the model was unchanged from the main model. 


\subsection{Code Considerations}

The initial calculations were conducted on DYNA2D which is a Lagrangian, explicit hydro code. After a few attempts to simulate the China Lake experiments by shortening the time scale and using additional strategies to improve the time step, it was found that the calculation did not proceed fast enough to make explicitly based computation of the problem immediately convenient. Calculations were rather long running and a lot of experimentation seemed to be required to determine a suitable set of parameters that would permit more efficient running. We, therefore, changed to NIKE2D, a Langragian, implicit code, as a convenient tool for the purpose of conducting this feasibility study.

Our considerations regarding the choice of codes for the possible Phase 3 follow-on to this work include the following. NIKE2D (Ref. C) is an implicit Lagrangian hydro code that permits large time steps and it has a material model that is compatible with DYNA's model but simpler. Generalization of the material model in NIKE2D is likely to be more complicated than in DYNA2D because of NIKE's close coupling of the material model and the implicit time stepping. From the standpoint of ease of model development, therefore, it would be desirable to use DYNA.

For shorter time scale problems than the China Lake experiments (time scale of minutes) such as press loading and launching the rounds, the following approach is suggested. Time dependent bench mark calculations for short time scale problems using the model capabilities of NIKE can be performed after some additional work of establishing convergence tolerances and time step optimization. This is needed to help overcome at least one known problem and to smooth some of the solutions. An effort could then be made with DYNA, using the identical material model, to replicate these results and to search for techniques that improve the time step. If successful, this could lead to a set of control parameters that need to be used for no purpose other . than to assure acceptable DYNA performance for this general class of problems. Additional model development could then be performed in the context of DYNA2D. If the effort to find such a set of parameters that make the application of DYNA to this project convenient is unsuccessful, the needed additional model development can be done in NIKE, although at somewhat greater expense.

\subsection{Computing}

A China Lake experiment was set up on NIKE2D for simulation. The dimensions were chosen to duplicate the essential experimental dimensions and clearances (Fig. 1); calculations were done on a time scale comparable to the experimental time scale (order 100 seconds). Slide lines were used 
between all material interfaces. We note that steel deformation caused no contact between the pistons and the cylinder walls

A number of calculations were performed to validate the code and to assess its behavior at our low stress levels. For this purpose the sample was represented by a perfectly elastic material that had a bulk modulus 0.01 times that of steel. It was determined that along slide lines good zoning matches were needed to somewhat suppress noise in the stress field in the sample at the sample-cylinder interface. Along slide lines special "augmentation" algorithms (fictitious springs that let the slide lines close smoothly) had to be invoked to prevent slide line crossing etc. By and large NIKE was shown to be capable of calculating the full compression and release cycle although premature code crashes were encountered.

The necessary force balances were investigated in the computer model and were found to satisfy physical requirements. This includes the direct transmission of force from the top piston to the bottom piston via the sample in the absence of friction. When friction is enabled, only some of the applied force is transmitted to the bottom piston with the balance being transmitted to the cylinder via wall friction between the sample and the cylinder wall.

However, several caveats are in order. They include an anomaly which is believed to be a consequence of the mismatch in the elastic moduli of the sample and the steel walls. It manifests itself by contracting the cylindrical boundary of the sample surface when the sample is first loaded axially by the pistons. This is clearly nonphysical and disappears when the sample is loaded further. A possible "fix" would reduce the time step during the early phases of the loading and some experimentation with code tolerances is also desirable to possibly alleviate this problem. Presumably such a strategy would also forestall premature problem crashes. It is believed that similar strategies can reduce calculational noise as well. Experiments in this area were not conducted in any depth.

\subsection{Results and Discussion \\ 2.7.1. Without Friction}

Two calculations of experiments on RS41 of the type conducted by China Lake are reported here. A third calculation is mentioned only briefly. The first calculation (dubbed rs10) simulated the compression of RS41 in the absence of all friction. Fig. 5 shows the computational mesh at maximum compression. The slight misalignment of the vertical meshlines running through the pistons and the sample stems from the fact that the same initial clearance existed between the sample and the wall as between the pistons and the wall. In the figure, the sample has distorted to fill this gap. Figures $6 \mathrm{~A}-6 \mathrm{C}$ show the maximum, minimum and differences in the principal stresses. The 
symmetry is reassuring and suggests that there are no end effects in the experimental apparatus. Fig. 7 shows the mesh after piston withdrawal. No problem anomalies are observed. Fig. 8 shows the radial stresses at the same time. The stress levels are seen to range up to $20 \mathrm{bars}$ compared to the maximum applied stresses of order $1 \mathrm{~kb}$. These stresses are the result of the elastic properties of the compacted powder. The choppiness in the contours at the sample-cylinder interface is computational noise which might be alleviated by refinement in the zoning and by other strategies alluded to above.

Figures $9 \mathrm{~A}-9 \mathrm{C}$ show stress profiles at maximum compression. The profiles are taken along axial lines of the computational model at regular radial intervals. Fig. 9A shows that the radial stresses are compressive and constant at about $0.50 \mathrm{~kb}$ throughout the sample (the "square well" profiles) and decay continuously throughout the cylinder. Figure $9 \mathrm{C}$ shows a similar behavior for the hoop stresses. Fig. 9B shows that axial stresses deviate only slightly from uniformity through the pistons and the sample. The saw teeth on the lower curves are probably associated with a slight Poisson effect in the steel pistons.

This calculation without friction was used to determine the relationship between the hoop strain of the outside of the cylinder and the radial stress in the sample. The resulting curve along with extrapolated linear and quadratic fits are shown in Fig. 10. This inferred data is important because China Lake measured these hoop strains from which we can now deduce the radial stresses in the sample using this calculated data. It is important to note that this method of stress inference yields stresses that are more than four times as large as those obtained from an analytic formula for a thick walled, infinite cylinder loaded along its entire length. A nonlinear reduction of this - the . only triaxial data for RS41 - is, therefore, deemed to be essential.

\subsubsection{With Friction}

The second calculation (dubbed rs11) that we report on was in every way identical to the previous calculation except that friction was enabled at the sample-cylinder interface. The force balances satisfy all the physical requirements. The coefficient of friction was assumed to be constant and was set to 0.25 . This apparently high value will be justified later.

Fig. 11 shows the computational mesh at the time of maximum compression. The distortion of the mesh compared to the problem without friction (Fig. 5) is immediately obvious. A check of the alignment of the vertical mesh lines reveals an appreciable amount of sliding on the piston faces. The early opening of a gap (numerically induced) between the sample and the cylinder wall does not seem to be damaging to the calculation because of the absence of friction on the piston faces. This enables the sample to slide freely towards the cylinder wall upon further loading. Fig. 12 shows contour lines of the 
maximum displacement in the sample at maximum compression. Comparison with Fig. 12A, obtained from the no-friction case at the same time, clearly shows the dragging on the walls that results from friction.

Fig. 13 shows the axial stress profiles along the same axial lines as above through the pistons and the sample after piston withdrawal. Friction, which holds the sample in place, causes the sample to apply a stress of about 20bars to the lower piston. Fig. 14 shows the contours of residual excess compression in the sample at the same time. The variation in the range of excess compression is about $17 \%$. This excess compression is indicative of the final density distribution in the sample and will be altered only slightly by removal of small external stresses exerted by the side wall of the experimental apparatus after piston withdrawal. By comparison, no appreciable density gradients are found in the sample in the absence of wall friction. In fact, the excess compression in the absence of friction (run rs10) at the same time is found to be 0.703 . with a total deviation of $0.3 \%$.

A third problem was attempted that was like the above problems but friction between the piston faces and the sample was enabled. Since motion in this direction is very limited, the results (up to an untimely crash) were not much different in character from the previous result. The presence of the anomaly of the sample's early radial contraction under loading apparently introduces only a minor perturbation.

A problem (dubbed rs14) was run that differed from problem rs11 only in that the deviatoric stress component on the crush curve was one sixth of the total stress as already discussed. This choice of values for the tensorial portion of the model makes the material more fluid-like. Fig 14A shows the computational mesh at maximum compression to be significantly more distorted than the corresponding mesh shown in Fig. 11. Fig 14 shows the maximum displacements to be greater than those shown in Fig 12. In short, reducing the strength of RS41 permits much greater distortions to occur in even this idealized compaction geometry when wall friction is present.

\subsubsection{Problem Comparison}

Fig. 15 summarizes the effect of friction on the area-averaged axial stresses on the pistons. Without friction the applied stress and the transmitted stress are calculated to be the same value. Since the cross sectional area of the pistons is assumed not to change, the associated axial forces are also the same. This, of course, is the expected result. It should be noted that the compacted sample pushing on the cylinder induces a distortion that causes some amount of axial stress in the cylinder. This stress, however, is symmetric about the sample mid plane and, therefore, makes no net contribution to the axial force balance on the cylinder. The symmetry of stresses was noted in the discussion of Fig. 6A-C above. 
When friction is included, a given piston displacement induces an applied stress of greater magnitude than without friction. The magnitude of the corresponding transmitted stress decreases compared to the no-friction case. These two stresses, for the case with friction, bracket the values for the case without friction. It appears then that the effect of friction at a given sample compression is to separate the values of the applied and transmitted stresses that are found when friction is absent. The magnitude of the applied stress is driven to a higher value and that of the transmitted stress is driven to a lower value. This separation of stresses appears to increase with increasing friction forces.

Fig 15A gives the corresponding results for problem rs14 in which the axial stress on the crush curve is partitioned to favor more nearly isotropic material behavior. Also shown is the computed average radial stress in the sample. It is interesting to note that the applied and transmitted stresses are essentially unchanged from problem rs11 (Fig 15) despite the calculated internal differences in the sample. The apparent decay in the applied stress unfortunately appears to be numerically induced and is linked to a "creeping" of the stress contours when the sample is at constant volume. This phenomenon cannot be attributed to any aspect of the model.

\subsubsection{Comparison with Experiment}

Fig. 16 compares our calculation with an experiment (China Lake's COMPAC32) whose results provided some of the input for our model definition. The piston displacement in the calculation is seen to be only very slightly less than in the experiment (dashed lines) so that the boundary conditions in the simulation and in the experiment are substantially the same. By comparing the applied stresses and the transmitted stresses we see that our prototype model for RS41 gives results that are similar to the experiment. The most obvious difference is the lesser separation between the applied and the transmitted stresses that are calculated by the model as compared to the experimental data. As noted above, increasing the importance of the friction term will very likely increase this spread.

Also shown are the computed, average radial stress that prevails in the sample and the radial stress that is inferred from the experiments as discussed earlier. To the extent that this data is deemed to be reliable, the calculation overestimates this quantity by nearly a factor of three. Comparing this computed radial stress with that given in Fig 15A for problem rs14 we see that these two values can be brought into closer coincidence (should this be justified by further experimental validation) by increasing the importance of the tensorial component of the crush curve. This would make the material behavior of RS41 more similar to that of a solid. 
On the subject of friction we would like to add a parenthetical comment. From the applied and transmitted stresses and from the piston displacements, we inferred the axial traction stress which is normalized to the area of the cylinder wall that is exposed to the sample. The input data from which these inferences were drawn is all considered to be reliable data. The ratio of this traction stress to the radial stresses in the sample should be the coefficient of friction. As discussed earlier, this latter stress is based on much less reliable data. However, it may be useful to note, as a way of bounding the problem, that the implied coefficients of friction range up to about 0.5 for applied stresses that reach about $0.5 \mathrm{~kb}$ while 1.0 appears to be the limit for the higher stress experiments where the applied stress reaches $1.5 \mathrm{~kb}$. We mention this very tentative observation because it appears to support the need for higher friction forces to achieve the greater separation of applied and transmitted stresses than are calculated with the prototype model.

The level of the stress average can be increased by "stiffening" the model. This is justified for the following reason. In the model formulation the mean of the applied and transmitted stresses along with the total average compression in the bed were used to define the state. (We referred to this as linear data interpretation above.) As noted earlier, substantial portions of the sample are compressed beyond this average value and therefore, operate in a domain of the model which is the result of extrapolation. In this prototype model this extrapolation is a completely arbitrary one.

A slight difference in the slope of the release between experiment and calculation is also noticeable. An improved fit is possible by generalizing the fitting function for the bulk modulus applicable to the release from just a constant value. It should be noted that the data set that we worked with was constructed by China Lake by just sampling their entire data set which consists of thousands of points for each curve. A more elaborate model treatment and data reduction effort would use their entire data set.

\subsection{Model Extensions}

The China Lake data consistently show a relaxation of stresses in the sample that occurs over time at constant volume (central plateau in Fig. 16). This phenomenon can not be explained as simply air escaping from the sample because the void space in the powder is compressed only about tenfold. The implied pressure change in the sample accounts for only 10 bars or order $1 \%$ of the total stress. The observed stress relaxation in the experiment, however approaches $10 \%$. A phenomenology that is a candidate to help model the stress relaxation is creep.

For Phase 3 we recommend the extension of the pore crush model in NIKE with such a time dependent capability. Creep already exists elsewhere in NIKE 
and its inclusion in the pore crush model might thus be facilitated. Creep necessarily occurs on a rather long time scale and it interacts with the pore crush nonlinearly. A benchmark comparison may, therefore, be needed to support efforts to improve the time stepping in DYNA when creep is added to its material model. Such a model is likely to have significant consequences on characterizing the long term behavior of stored rounds that are loaded with RS41.

At this time NIKE permits only a constant bulk modulus for the unloading from the compressed state. China Lake's data includes release data from two different stress levels: $0.5 \mathrm{~kb}$ and $1.2 \mathrm{~kb}$. No clear need for compression dependent release moduli is apparent from this data. However, an additional degree of freedom in this variable would probably permit a more precise match to the curvature of the release path.

As part of Phase 3 some numerical experimentation also needs to be done to explore the settings of convergence and other tolerances to optimize NIKE's calculational ability for both accuracy and efficiency for this type of problem. Among other things, the phenomenology of tensile strength needs to be fully evaluated in the context of NIKE2D. The implications of using DYNA2D instead of NIKE2D as the main simulation have been discussed earlier.

\section{Conclusions}

We have made a demonstration of the consistency of China Lake's data for RS41 and have constructed a prototype model for this material. The calculation of a simple double-piston experiment with this model clearly demonstrates that compression of RS41 results in permanently embedded density gradients when wall friction is present and is in reasonable agreement with the observed behavior characteristics of the powder.

We have further demonstrated that NIKE2D with some amount of numerical experimentation and additional model development will likely be able to calculate the results of mechanically working this material. To support further development, additional experiments are recommended in Phase 3 to refine the data on the frictional and the strength and hydrostatic properties of RS41. Further numerical study is needed to determine whether such data can be inferred from measurements taken on some simulant material. Thus, we believe that the feasibility of the approach has been established and recommend proceeding with the Phase 3 model development.

The calculations described here focussed on the first of the proposed calculations, the double piston experiments at China Lake. The extent of the numerical analysis, data analysis, and comparison of data with numerical results were felt to be sufficient to establish the feasibility of the powder 
model for additional development and refinement under Phase 3. The major features of the model that the second proposed calculation was to demonstrate, namely the existence of axial density gradients due to press loading, were in fact observed in the double piston calculation. Also, the programmed level of effort for Phase 2 calculational assessment was fully expended on the double piston analysis.

The experimental tests proposed by LLNL were not able to be performed under this effort, because of personnel changes during the period of performance. However, an improved plan for generation of important powder characterization data will be proposed in Phase 3, with appropriate cost adjustments.

These issues are discussed in more detail in the Phase 3 Proposal, under separate cover. 


\section{Acknowledgment}

We thank Chan Price and Alice Atwood of China Lake for making their data available to us and for their cooperation in resolving data inconsistencies.

We also thank Peter Raboin of LLNL for helpful suggestions and comments in the use of NIKE2D. A code repair was also greatly appreciated.

\section{References}

Ref. A

W.L. Elban and M.A. Chiarito, Quasistatic Compaction Study of Coarse HMX Explosive, Powder Technology, Vol. 46, No. 2-3, pp. 181-193

Ref. B

Chan Price, Alice Atwod, private communications, (1995)

Ref. C

Bruce Engelmann, J.O Hallquist, Originator, NIKE2D, A Nonlinear, Implicit, Two-Dimensional Finite Element Code for Solid Mechanics,

User Manual (April 1991)

Ref. D

T.A. Laursen and B.N. Maker, An Augmented Lagrangian Quasi-Newton Solver for Constrained Nonlinear Finite Element Applications, Int. J. Num. Meth. Eng., (to appear), (1995) 


\section{Appendix}

This appendix contains the documentation of the material model. First we give a short description of the physics of the model. This is followed by examples of the numerical input to MAZE and NIKE2D for the prototype model that was employed in the calculation dubbed rs11. Also included is the input for the "Lagrange augmentation" which is needed for accurate slide line treatment as mentioned below.

The basic model includes the phenomenon of pore crush with a perfectly elastic release from a compressed state. Reloading material that is in a partly compacted state occurs by loading elastically until the crush curve is reached from where material crush resumes upon further loading. An example of a loading-unloading curve is shown in Fig. A. NIKE2D accepts a numerically defined crush curve of pressure versus the negative of volumetric strain. NIKE allows ten points for this purpose. The unloading-reloading is governed by a constant bulk modulus for all states of compaction.

An elastic, perfectly plastic strength model is combined with the pore crush model. The shear modulus is a constant for all states of material compression. The yield strength, $Y$, is defined analytically as a three parameter function of the pressure, $P$, as follows.

$$
Y=\left[3\left(a_{0}+a_{1} P+a_{2} P^{2}\right)\right]^{1 / 2}
$$

Here $a_{0}, a_{1}$ and $a_{2}$ are constants. All inputs to the pore crush and strength model are available through MAZE as seen in the attached example. The resulting MAZE output that serves as NIKE input is also shown.

NIKE2D input that is not available through MAZE is Lagrange augmentation. The augmentation procedure (fully documented in Ref. D) assures accuracy of the slide-line constraints within user specified tolerance, independent of penalty stiffness value. This option was found to be essential to obtain proper code performance. This input has to be set by hand in the NIKE input file and the settings and placement relative to the slide line definition input lines is shown in the NIKE input example. Successful setting of these variables is reported in the NIKE print file. An example is included. 


\section{MAZE Input}

c model for rs $41 \quad \ldots$ epsvol $=\ln (\mathrm{V} / \mathrm{V} 0)$

mat 45

r0 1.0688

g $0.009 \mathrm{ku} 0.018$ a0 1e-10 a1 0.0 a2 0.1875 pc-1.0e00 ul 0 npts 10

vs

0.000000e+00 $\quad 9.945455 \mathrm{e}-02 \quad 1.899962 \mathrm{e}-01 \quad 2.730167 \mathrm{e}-01 \quad 3.496698 \mathrm{e}-01$ 4.208635e-01 $\quad 4.873233 \mathrm{e}-01 \quad 5.496406 \mathrm{e}-01 \quad 6.083010 \mathrm{e}-01 \quad 8.971619 \mathrm{e}-01$ $\mathrm{p}$

$0.000000 \mathrm{e}-00 \quad 3.774529 \mathrm{e}-06 \quad 2.554898 \mathrm{e}-05 \quad 6.909238 \mathrm{e}-05 \quad 1.388289 \mathrm{e}-04$ 2.420736e-04 3.904694e-04 6.020222e-04 9.039507e-04 3.000000e-03 endmat 


\section{NIKE2D Input}

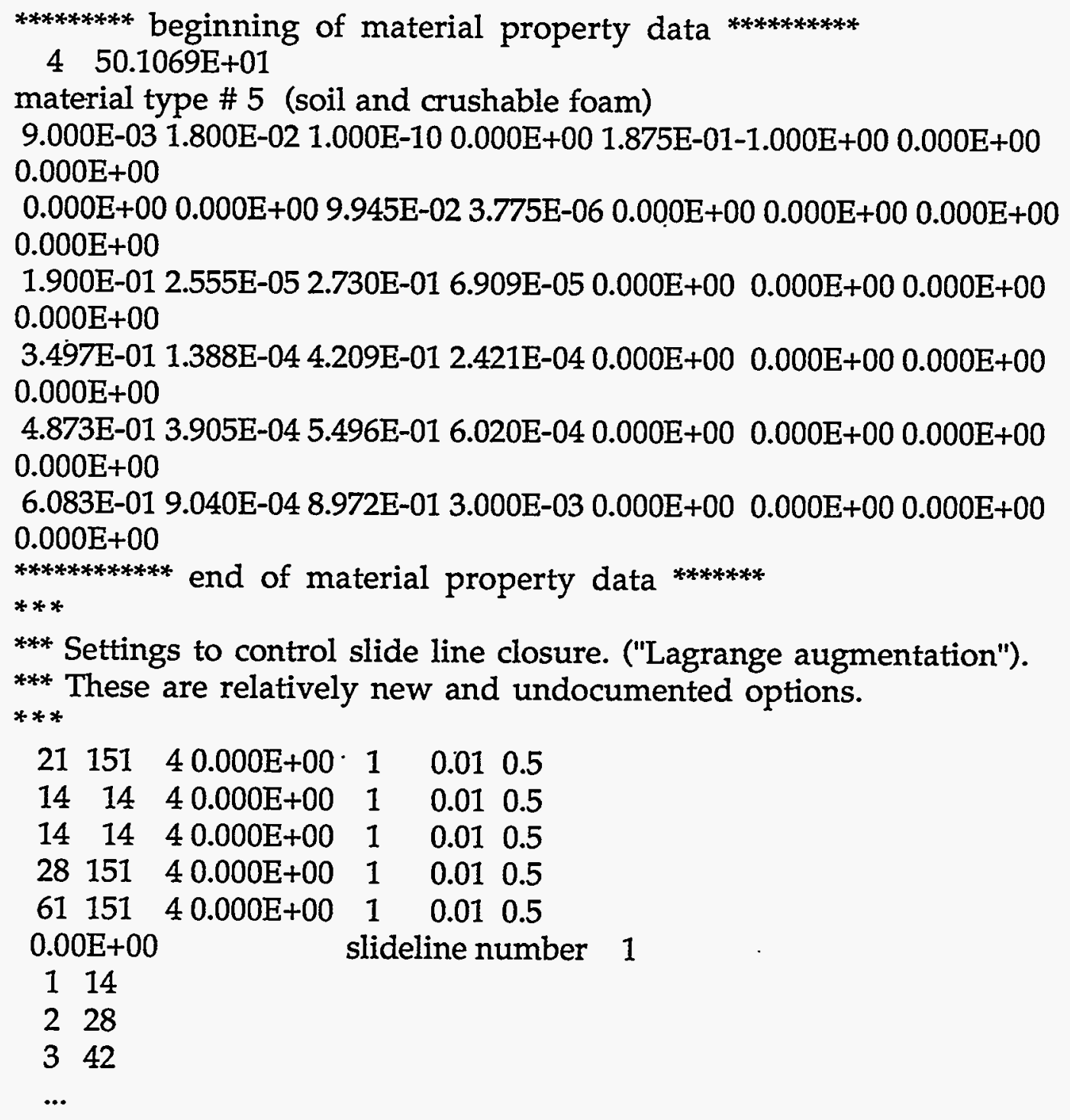


slide-line number 1

\# of slave nodes $=21$

\# of master nodes $=151$

slide-line type $=4$

stiffness scale factor $=0.100 \mathrm{E}+01$

lagrange augmentation flag 1

eq.0: no augmentations

eq.1: augmentations (conv. based on mult.)

eq.2: augmentations (conv. based on gap)

1t.0: fixed number of augmentations

augmentation tol. (normal dir.) $0.100 \mathrm{E}-01$

augmentation tol. (tangential dir.) $0.500 \mathrm{E}+00$

small penetration flag 0

eq.0: all penetrations considered

eq.1: only small penetrations considerd 


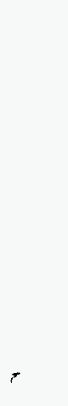





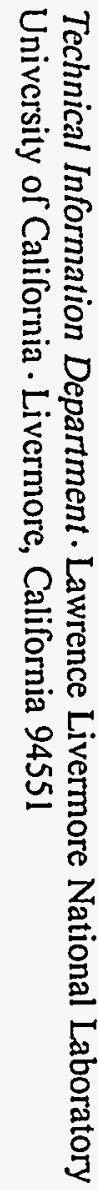

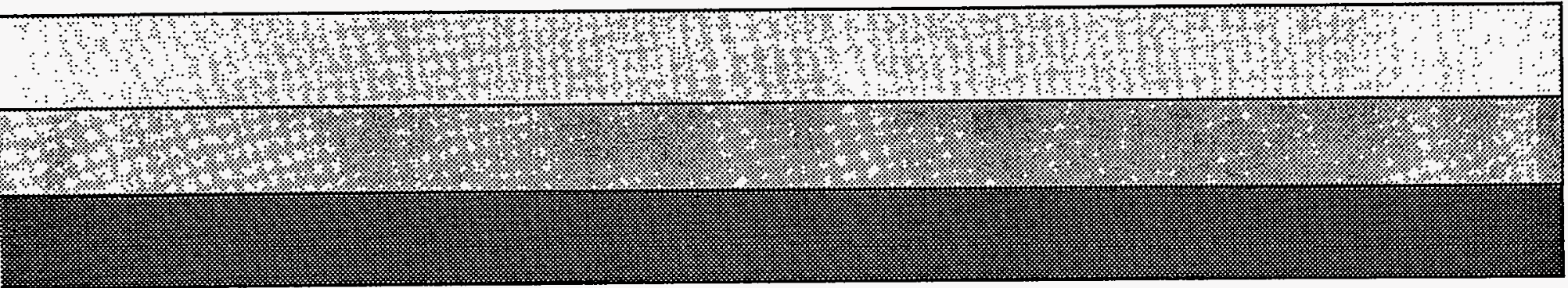

